\title{
Specific correlation effects in inner-electron photoelectron spectroscopy
}

\author{
Donald R. Beck \\ Physics Department, Michigan Technological University, Houghton, Michigan 49931
}

C. A. Nicolaides

Theoretical and Physical Chemistry Institute, National Hellenic Research Foundation, 48 Vas. Constantinou Avenue, Athens 501/1, Greece

(Received 8 September 1981; revised manuscript received 14 December 1981)

\begin{abstract}
We show that the large discrepancies between single- and multiconfigurational DiracFock calculations and experiment recently obtained for certain binding and Auger energies of $\mathrm{Ar}, \mathrm{K}, \mathrm{Kr}, \mathrm{Rb}, \mathrm{Sr}$, and $\mathrm{Xe}$, can be removed by including easily computed symmetric-exchange-of-symmetry correlations.
\end{abstract}

\section{INTRODUCTION}

Currently, photoelectron spectroscopy (PES) yields accurate information about one-electron binding energies (BE) and Auger energies (AE) and transition probabilities in atoms, molecules, and solids. ${ }^{1}$ The rigorous calculation of these quantities involves the consideration of relativistic, radiative, and correlation effects (see Refs. 2-4 and references therein). Although the magnitude of the first two increases rapidly with the nuclear charge $Z$ or degree of ionization, the importance of correlation effects in interpreting, for example, atomic or solid-state photoelectron spectra is not eliminated, especially for valence- and intermediate-shell photoexcitation processes throughout the periodic table.

The purpose of this paper is to present new results of calculations on certain a priori chosen electron correlations which perform the following:

(1) remove the existing large discrepancies between certain previous Dirac-Fock ${ }^{5,6}$ (DF) and multiconfigurational Dirac-Fock ${ }^{7}$ (MCDF) calculations of $\mathrm{BE}$ and $\mathrm{AE}$ and experimental values from photoelectron spectra of $\mathrm{Ar}, \mathrm{K}, \mathrm{Kr}, \mathrm{Rb}, \mathrm{Sr}$, and $\mathrm{Xe}$;

(2) support previous suggestions $\mathrm{s}^{2-4,8-15}$ that for

(a) binding and Auger energies,

(b) radiationless and radiative transition probabilities in the discrete or the continuous spectrum, and

(c) semiquantitative predictions of photoelectron spectra (e.g., appearance of satellite peaks), a few specific and easily identifiable correlation effects play an exclusively dominant role beyond the
Dirac-Fock (or Hartree-Fock, nonrelativistically) approximation.

Thus, these results add to the accumulating evidence that the reasonable interpretation and prediction of inner-shell photoionization spectra need not involve extensive and complex calculations, provided one computes the wave functions and corresponding energy differences at the Dirac-Fock plus specific correlations $s^{2-4,10}$ level. Furthermore, once these effects are taken into account, solid-state bulk ${ }^{3,15,16}$ and surface ${ }^{17}$ perturbations can also be computed efficiently for metals or ionic compounds, thus providing a more or less complete description of inner-shell ionization in atoms and many solids.

\section{MOST IMPORTANT CORRELATION EFFECTS FOR TRANSITION ENERGIES
AND PROBABILITIES}

Binding or Auger energies are rigorously defined in terms of differences of total energies of stationary or nonstationary states ${ }^{3,11-13}$ assuming the result does not depend strongly on the dynamics. ${ }^{3}$ If these energies are broken down in terms of different contributions, the BE's (and AE's) can be expressed as follows ${ }^{3}$ :

$$
\mathrm{BE}=\Delta E_{\mathrm{DF}}+\Delta E_{\mathrm{corr}}+\Delta E_{\mathrm{rel}}+\Delta E_{\mathrm{rad}},
$$

where we have the following.

$\Delta E_{\mathrm{DF}}=$ the energy difference between two self-consistent field (SCF) DF calculations.

$\Delta E_{\text {corr }}=$ the correlation energy difference for 
the initial and final states.

$\Delta E_{\text {rel }}=$ the difference between the relativistic effects-beyond the Hamiltonian used for DiracFock-using an appropriate relativistic interaction operator (e.g., Breit).

$\Delta E_{\mathrm{rad}}=$ the difference between the radiative corrections.

The theory and method of calculation of $\Delta E_{\mathrm{DF}}$, $\Delta E_{\mathrm{rel}}$, and $\Delta E_{\mathrm{rad}}$ (for $s$ electrons) in many-electron systems can be found in Refs. 3, 7, and 18-21.

$\Delta E_{\text {corr }}$ represents the contribution of electron correlation. Its exact magnitude depends on the type of transition and system under examination. The main conceptual and practical disadvantage of wave-function methods which aim at the calculation of total energies, is that a part of electron correlation (EC), which contributes to both initial and final states, is computed twice and then subtracted out for the calculation of BE. Thus, a useful and efficient theory of BE should predict those correlations which, to a good approximation, cancel upon subtraction and those which do not and give rise to the conspicuous deviations from the predictions of the Dirac-Fock (Hartree-Fock) theory regarding radiationless and radiative transitions in PES. As a simple example of this statement, let us consider the $1 s \mathrm{BE}$ of $\mathrm{Be} 1 s^{2} 2 s^{2} S$. Suppose we write $H=H_{0}+V$, where $H_{0}$ is separable, and obtain a one-electron basis set of energies $\epsilon_{n}$ from $H_{0}$ which we use for initial and final states. Then, the $2 s^{2}$ electron correlation to second order is given by

$$
\begin{aligned}
E C_{1 s^{2} 2 s^{2}}\left(2 s^{2}\right) & \\
= & \oiint_{n, k, l} \frac{\left.\left\langle 1 s^{2} 2 s^{2}|V| 1 s^{2}[n(k) l]^{2}\right\rangle\right|^{2}}{2 \epsilon_{2 s}-2 \epsilon_{n(k) l}}, \quad \text { (1) } \\
E C_{1 s 2 s^{2}}\left(2 s^{2}\right) & =\bigoplus_{n, k, l} \frac{\left\lfloor\left.\left\langle 1 s 2 s^{2}|V| 1 s[n(k) l]^{2}\right\rangle\right|^{2}\right.}{2 \epsilon_{2 s}-2 \epsilon_{n(K) l}} \\
& +\underbrace{}_{n, K} \frac{\left\lfloor\left.\left\langle 1 s 2 s^{2}|V| 1 s^{2} n(k) s\right\rangle\right|^{2}\right.}{2 \epsilon_{2 s}-\epsilon_{1 s}-\epsilon_{n(k) s}},
\end{aligned}
$$

where $n$ and $k$ stand for the discrete and continuous spectrum energy parameters correspondingly. The first term of Eq. (2) cancels that of Eq. (1). The second term of Eq. (2) describes hole-filling correlations (here the hole is in the $K$ shell) which contributes both to the autoionization width and energy shift (see Refs. 22, 11, and 12 for theory and early applications to inner-hole states of light atoms).

In Refs. 2-4 we have presented a theory of electron correlation in ground and excited states as well as radiative (and radiationless) transition probabilities (see also Refs. 15, 22, and 23) which is based on an analysis of EC from a subshell cluster expansion of the wave function. It has been applied to problems of PES quantitatively as well as semiquantitatively. Its main characteristics are (the reader is referred to Refs. 2-4 for a complete exposure, examples, and other references) the following.

(1) The zeroth order is, in general, a multiconfigurational vector which includes those valence configurations which mix heavily and affect the overall character of the wave function the most, together with the symmetric-exchange-of-symmetry (SEOS) correlations to be defined below. We have called this vector the Fermi-sea (FS) vector. ${ }^{8,2}$ The function space spanned by the orbitals orthogonal to the FS orbitals is called virtual. For states involved in inner-shell ionization, the corresponding angular symmetries of the subshells entering the Fermi-seas are usually the same. They often play the major role in multiplet and satellite structure in PES. $3,4,10$

(2) Given the way they are chosen, Fermi-sea orbitals are strongly state dependent. $N$-electron correlation configurations which contain at least one new subshell belonging to the FS affect the wave function the most. Pair correlation functions which are orthogonal to the FS orbitals and do not contain any FS orbitals are, to a very good approximation, independent of the symmetry and shell structure of the zeroth-order single-configurational vector. Therefore, the significance of these bivirtual correlations is mainly energetic and to a much lesser degree spectroscopic_although indirectly they may sometimes have an effect on the energy positions and mixing coefficients of spectroscopic configurations. ${ }^{24}$ The calculation of the symmetrized bivirtual pair functions which survive after subtraction of initial- and final-state bivirtual pair energies $^{3}$ is carried out variationally, first independently and then from a total configurationinteraction calculation. ${ }^{2}$

(3) Apart from the Fermi-sea and the surviving bivirtual correlations, there are two very specific correlation effects, one of which (b), if it is present, causes the most extreme changes in the wave func- 
tions of core excited states. ${ }^{3,15}$ These are

(a) single orbital excitations of the type

$$
n l \leftrightarrow(l \pm 2),
$$

where $n l$ is the occupied Hartree-Fock orbital of $l$ symmetry and $(l \pm 2)$ is the symmetry of the interacting Fermi-sea or virtual orbital (e.g., $n s^{2} n p$ $\leftrightarrow n s n p n d$ or $d \leftrightarrow s$ interactions in the transition metals).

(b) SEOS correlations. These are the correlation effects which are singled out in this and previous papers as being the main cause of the deviation between single- or multiconfigurational Dirac-Fock computations and certain experimental results. They are recognized in terms of the orbital symmetries of Fermi-sea and virtual orbitals:

$$
\left(n l, n l^{\prime}\right) \leftrightarrow\left[(l-1),\left(l^{\prime}+1\right)\right] \text { SEOS },
$$

where $n l$ are the occupied HF orbitals, $(l-1)$ is the symmetry of the hole, and $l^{\prime}+1$ is the symmetry of a Fermi-sea or virtual orbital. If allowed, excitations into only Fermi-sea empty orbitals are always more important (e.g., $\mathrm{Mg} 3 p^{21} D \leftrightarrow 3 s 3 d^{1} D$ ).

The importance of SEOS correlations was discovered during the course of our early work on transition probabilities ${ }^{25,26}$ and autoionizing states, ${ }^{22}$ where electron correlation was analyzed according to Sinanoğlu's theory. ${ }^{27,28}$ Their role was pointed out explicitly in Ref. 8 in connection with spectra involving mixings of configurations of the type $p^{2} \leftrightarrow s d, d^{2} \leftrightarrow p f, p d \leftrightarrow s f$.

We point out that Fermi-sea and SEOS correlations correspond to what other researchers have independently studied and called, for example, "initial- (final)-state configuration-interaction"29 "super Coster-Kronig spectra", ${ }^{30}$ etc.

In Refs. 3 and 15 we have presented the above facts as general rules and heuristic or simple computational tools applicable to the interpretation of photoelectron spectra throughout the periodic table, with specific examples and predictions.

In this paper we present the first explanations of the large discrepancies (or lack of them) between theory and a number of recent experimental values-even when the theory has been carried out at the multiconfigurational Dirac-Fock level ${ }^{7}$-in terms of SEOS.

\section{APPLICATIONS AND RESULTS}

In this work, all electron correlation effects are treated at the nonrelativistic level. While a rela- tivistic treatment would be preferable, particularly for heavy atoms such as Xe, it is still difficult to do using existing methods, such as multiconfiguration DF, principally due to the increased number of $N$-electron functions needed when $j j$ coupling is used; i.e., one needs to include all relativistic configurations which reduce, in the $c \rightarrow \infty$ limit, to the important nonrelativistic SEOS configurations.

The method used to compute SEOS is variational and follows the theories described in Refs. 2 and 23. Since, in this work, we are after only energy shifts (and not cross sections or widths), no explicit use of continuum orbitals is made.

The results of our computations are presented in Tables I and II. Below they are analyzed briefly in order to illustrate the general observations of the previous sections.

\section{A. BE of $3 p$ in $\mathrm{Kr}$}

The ground-state configuration of $\mathrm{Kr}$ is $K L M 4 s^{2} 4 p^{6}$. After the removal of a $3 p$ electron, the excited-state single configuration is $K L 3 s^{2}$ $3 p^{5} 3 d^{10} 4 s^{2} 4 p^{6}$. As with all cases of inner-hole excited states, the valence Fermi-sea configurations are the same for initial and final states. The most important wave-function and energy effects are caused by the SEOS correlations in the excited state: $3 d^{2} \leftrightarrow 3 p f, 3 s 3 d \leftrightarrow 3 p f$. For the ground states of the rare-gas and alkali atoms considered here, the single orbital excitations do not affect the BE. For the excited state, the only important single excitation is $3 p \rightarrow f$. Thus, it is seen that in the $\mathrm{Kr}$ excited state, Fermi-sea $(4 f)$, and virtual orbitals of $f$ symmetry are important. When bivirtual correlations are considered, the only contributors to the $\mathrm{BE}$ turn out to be the ground-state excitations which are formed from the $3 p^{2}, 3 s 3 p$, and $3 p 3 d$ pairs. Here, the bivirtual correlation effects which survive cancellation increase the BE.

\section{B. Prediction of $2 p_{1 / 2}, 2 p_{3 / 2}$ BE of $\mathrm{K}$. Absence of SEOS correlations}

Recently, Aksela et al. ${ }^{31}$ reported accurate measurements of the $2 p_{1 / 2}$ and $2 p_{3 / 2}$ BE's in $\mathrm{K}$. These are $303.6 \pm 0.1 \mathrm{eV}$ and $300.7 \pm 0.1 \mathrm{eV}$, respectively. They compared them with the theoretical DF values of Huang et al. ${ }^{6}$ which are 303.0 and $300.2 \mathrm{eV}$ correspondingly. Now we note that the absence of the strong SEOS correlations brings the DF results reasonably close to experiment-as is to 
TABLE I. Gas-phase binding energies (BE) in $\mathrm{eV}$.

\begin{tabular}{|c|c|c|c|c|c|c|}
\hline $\begin{array}{c}\text { Subshell/ } \\
\text { species }\end{array}$ & $\mathrm{DF}^{\mathrm{a}}$ & Corr $^{b}$ & Total theory $^{\mathrm{c}}$ & & Experimental & \\
\hline $2 p_{1 / 2} \mathrm{~K}$ & 303.0 & \multirow{2}{*}{0.7} & $303.7^{\mathrm{d}}$ & $303.6^{\mathrm{e}}$ & $303.2^{\mathrm{f}}$ & $303.7^{g}$ \\
\hline $2 p_{3 / 2} \mathrm{~K}$ & 300.2 & & $300.9^{d}$ & $300.7^{\mathrm{e}}$ & $300.5^{\mathrm{f}}$ & $300.9^{g}$ \\
\hline $3 p_{1 / 2} \mathrm{Kr}$ & $225.01^{\mathrm{h}}$ & \multirow{2}{*}{$-2.76^{i}$} & 222.3 & & $221.8^{\mathrm{j}}$ & \\
\hline $3 p_{3 / 2} \mathrm{Kr}$ & $217.03^{\mathrm{h}}$ & & 214.3 & & $214.2^{\mathrm{j}}$ & \\
\hline $3 p_{1 / 2} \mathrm{Rb}$ & $257.2^{\mathrm{h}}$ & \multirow{2}{*}{$-2.57^{\mathrm{i}}$} & 254.6 & & $254.3^{\mathrm{f}, \mathrm{h}}$ & \\
\hline $3 p_{3 / 2} \quad \mathrm{Rb}$ & $248.0^{\mathrm{h}}$ & & 245.4 & & $245.4^{\mathrm{f}, \mathrm{h}}$ & \\
\hline $3 p_{1 / 2} \mathrm{Sr}$ & $291.3^{k}$ & \multirow{2}{*}{$-2.50^{\mathrm{d}, \mathrm{i}}$} & 288.8 & & $289.1^{\mathrm{g}}$ & \\
\hline $3 p_{3 / 2} \mathrm{Sr}$ & $280.7^{\mathrm{k}}$ & & 278.2 & & $278.4^{\mathrm{g}}$ & \\
\hline
\end{tabular}

咅Dirac-Fock calculation using Desclaux's program (Ref. 18) with the Breit operator evaluated to zeroth order. Level splittings in the alkali's have been ignored due to their size $(<0.1$ $\mathrm{eV}$.

${ }^{\mathrm{b}}$ Correlation evaluated nonrelativistically.

${ }^{\mathrm{c}}$ The sum of footnotes $a$ and $b$, unless otherwise indicated (i.e., radiative effects not included).

${ }^{\mathrm{d}}$ From Ref. 3, Table III. There are no significant SEOS effects. This reference also gives $\mathrm{BE}$ for other $\mathrm{K}$ electrons, Cs electrons, and $1 s$ electrons of $\mathrm{Li}, \mathrm{Be}, \mathrm{B}, \mathrm{C}, \mathrm{O}, \mathrm{O}^{-}, \mathrm{F}, \mathrm{F}^{-}, \mathrm{Ne}$, $\mathrm{Na}$, and $\mathrm{Na}^{+}$.

${ }^{\text {e}}$ Reference 31 .

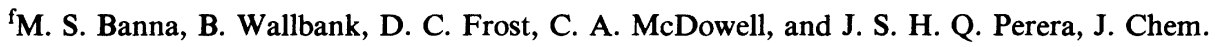
Phys. 68, 696 (1978); $\underline{68}, 5459$ (1978).

${ }^{8}$ Reference 32.

${ }^{\text {h}}$ Reference 5 .

iThis work.

jS. Svensson, N. Martensson, E. Basetter, P. A. Malmquist, U. Geluis, and K. Siegbahn, Phys. Scr. 14, 141 (1976).

${ }^{k}$ DF calculation was done by J. P. Desclaux and B. Fricke, cited in reference of footnote $\mathrm{g}$.

be expected. Nevertheless, there is still some remaining difference which is due to differential bivirtual ground-state correlation effects. These correlations were actually computed before the Aksela et al. ${ }^{31}$ measurements and the K BE were predicted to be 303.7 and $300.9 \mathrm{eV}$, in agreement with experiment (Table I).
C. $3 p_{1 / 2}, 3 p_{3 / 2} \mathrm{BE}$ in $\mathrm{Kr}, \mathrm{Rb}$, and $\mathrm{Sr}$

Recently, Key et al. ${ }^{5}$ have compared DF values to experiment for selected $\mathrm{BE}$ of $\mathrm{K}, \mathrm{Rb}, \mathrm{Cs}, \mathrm{Mg}$, $\mathrm{Zn}, \mathrm{Cd}, \mathrm{Ne}, \mathrm{Ar}, \mathrm{Kr}$, and $\mathrm{Xe}$ and, somewhat earlier, Mehlhorn et al. ${ }^{32}$ have done so for selected BE of Sr. The only significant $(>1 \mathrm{eV})$ discrepancies

TABLE II. Auger energies for rare gases (in eV).

\begin{tabular}{lrrrr}
\hline \hline Transition/species & $\mathrm{DF}^{\mathrm{a}}$ & Corr $^{\mathrm{b}}$ & $\begin{array}{l}\text { Total } \\
\text { theory }\end{array}$ & Experimental \\
\hline$L_{2}-M_{1} M_{1}{ }^{1} S_{0}$ in $\mathrm{Ar}$ & 168.4 & 10.4 & 178.8 & $180.1^{\mathrm{d}}$ \\
$M_{5}-N_{1} N_{1}{ }^{1} S_{0}$ in $\mathrm{Kr}$ & 19.6 & 8.7 & 28.3 & $30.9^{\mathrm{e}}$ \\
$M_{4}-N_{1} N_{1}{ }^{1} S_{0}$ in $\mathrm{Kr}$ & 20.9 & & 29.6 & $32.1^{\mathrm{e}}$ \\
$N_{5}-O_{1} O_{1}{ }^{1} S_{0}$ in $\mathrm{Xe}$ & 2.2 & 7.3 & 9.5 & $14.0^{\mathrm{f}}$ \\
$N_{4}-O_{1} O_{1}{ }^{1} S_{0}$ in Xe & 4.2 & & 11.5 & $16.0^{\mathrm{f}}$ \\
\hline \hline
\end{tabular}

${ }^{\mathrm{a} D i r a c-F o c k}$ results with some quantum electrodynamic effects from Ref. 7.

bStrong SEOS correlation computed nonrelativistically (this work).

'The sum of footnotes $a$ and $b$.

${ }^{d}$ W. Mehlhorn and D. Stalherm, Z. Phys. 217, 294 (1968).

${ }^{e}$ L. O. Werme, T. Bergmark, and K. Siegbahn, Phys. Scr. 6, 141 (1972).

fS. Ohtani, H. Nishimura, H. Suzuki, and K. Wakiya, Phys. Rev. Lett. 36, 863 (1976). 
found are for just those BE for which large SEOS correlations are expected. When these are included, together with the smaller differential bivirtual correlations, the agreement with experiment is quite satisfactory as Table I shows.

\section{D. $\mathrm{AE}$ in $\mathrm{Ar}, \mathrm{Kr}$, and $\mathrm{Xe}$ showing large SEOS correlations}

Recently, Beathem et al..$^{7}$ computed a large number of $\mathrm{BE}$ and $\mathrm{AE}$ for the rare gases using a multiconfiguration DF approach which included correlation effects of the Fermi-sea type but not of the SEOS type. Comparison with experimental values $^{7}$ shows that, for those $\mathrm{BE}$ and $\mathrm{AE}$ for which we would predict large SEOS effects, there is indeed a large discrepancy between theory and experiment.

Here, we choose to examine those AE's showing the largest discrepancies, i.e., the $n s^{\circ}$ final states $(n=3$ for Ar, $n=4$ for $\mathrm{Kr}, n=5$ for $\mathrm{Xe}$ ). These are expected to be the most troublesome as it is only for them that SEOS involves just FS orbitals. It is to be noted that the SEOS BE present in that work $^{7}$ also need to be corrected. In Table II, we see that the inclusion of SEOS correlation (double plus quadrupole excitations) removes most of the discrepancy, although our results deviate more as $Z$ increases, which probably indicates a relativistic treatment of correlation is needed for the larger- $Z$ species.

\section{E. SEOS in actinides}

Some results ${ }^{3}$ (references therein and this work) for strong SEOS effects in $n s(n \geq 3)$ and $n p$ $(n \geq 3)$ final-hole states are now available. A candidate from strong SEOS not yet explored are the $5 d$ binding energies in the actinides, for there the substitution $5 f^{2} \leftrightarrow 5 d 5 g$ is allowed (significant SEOS $4 f^{2} \leftrightarrow 4 d v_{g}$ will also be present for $4 d$ BE in the lathanides and above, but this should be weaker than for the $5 d$, as the $g$ orbital is virtual for them). Strong SEOS nonrelativistic calculations (this work and Ref. 33) for the $5 d$ hole state of nobelium lowered the $\mathrm{BE}-8.8 \mathrm{eV}$; an increase of nuclear charge to $Z=120$ (which pulls in the $5 g$ orbital, to make it "belong more clearly" to the $n=5$ shell) increased the lowering to $-11.9 \mathrm{eV}$. Observed anomalies ${ }^{34,35}$ are about one-half this size. While there are obviously other effects which should be incorporated (the remaining correlation treated relativistically, chemical shifts, etc.), it is clear that the above SEOS correlations cannot be neglected in any accurate treatment of such BE.

\section{ACKNOWLEDGMENT}

One of us (D.R.B) thanks Michigan Technological University for its support of a part of this work.
${ }^{1} X$-Ray Photoelectron Spectroscopy, edited by T. A. Carlson, (Dowden, Hutchinson and Ross, Stroudsburg, 1978).

${ }^{2}$ D. R. Beck and C. A. Nicolaides, in Excited States in Quantum Chemistry, edited by C. A. Nicolaides and D. R. Beck (Reidel, Boston, 1978), p. 105.

${ }^{3}$ D. R. Beck and C. A. Nicolaides, in Ref. 2, p. 329.

${ }^{4}$ C. A. Nicolaides and D. R. Beck, in Ref. 2, p. 143.

${ }^{5}$ R. J. Key, M. S. Banna, and C. S. Ewig, J. Electron Spectosc. Rel. Phenom. 24, 173 (1981).

${ }^{6}$ K. N. Huang, M. O. Aoyapi, M. H. Chen, B. Crasemann, and H. Mark, At. Data Nucl. Data Tables 18, 243 (1976).

${ }^{7}$ N. Beathem, I. P. Grant, B. J. McKenzie, and S. J. Rose, Phys. Scr. 21, 423 (1980).

${ }^{8}$ D. R. Beck and C. A. Nicolaides, Int. J. Quantum Chem. \$8, 17 (1974).

${ }^{9}$ D. R. Beck and C. A. Nicolaides, Int. J. Quantum. Chem. S10, 119 (1976).

${ }^{10}$ C. A. Nicolaides and D. R. Beck, Chem. Phys. Lett.
36, 79 (1975).

${ }^{11}$ C. A. Nicolaides, Chem. Phys. Lett. 19, 69 (1973).

${ }^{12}$ C. A. Nicolaides and D. R. Beck, Chem. Phys. Lett. 27, 269 (1974).

${ }^{13}$ D. R. Beck and C. A. Nicolaides, J. Electron. Spectrosc. Rel. Phenom. ㅈ, 249 (1976).

${ }^{14} \mathrm{G}$. Theodorakopoulos, C. A. Nicolaides, and D. R. Beck, Int. J. Quantum. Chem. S13, 671 (1979).

${ }^{15}$ D. R. Beck and C. A. Nicolaides, Int. J. Quantum Chem. S14, 323 (1980).

${ }^{16}$ D. R. Beck, Int. J. Quantum Chem. S15, 521 (1981).

${ }^{17}$ A. Andriotis and C. A. Nicolaides (unpublished).

18J. P. Desclaux, Comput. Phys. Commun. 9, 31 (1975).

${ }^{19}$ I. P. Grant, Adv. Phys. 19, 747 (1970).

20M. H. Mittleman, Phys. Rev. A 5, 2395 (1972).

${ }^{21}$ A. M. Desiderio and W. R. Johnson, Phys. Rev. A $\underline{3}$, 1267 (1971).

${ }^{22}$ C. A. Nicolaides, Phỵs. Rev. A $\underline{6}, 2078$ (1972).

${ }^{23}$ C. A. Nicolaides and D. R. Beck, Int. J. Quantum 
Chem. 14, 457 (1978).

${ }^{24}$ D. R. Beck and C. A. Nicolaides, Phys. Lett. $\underline{61 \mathrm{~A}}$, 227 (1977).

${ }^{25}$ D. R. Beck and O. Sinanoğlu, Phys. Rev. Lett. 28, 945 (1972).

${ }^{26}$ C. A. Nicolaides and D. R. Beck, J. Phys. B 6,535 (1973).

${ }^{27}$ H. J. Silverstone and O. Sinanoğlu, J. Chem. Phys. 44, 1898 (1966).

${ }^{28}$ I. Oksüz and O. Sinanoğlu, Phys. Rev. 181, 42 (1969).

${ }^{29}$ R. L. Martin and D. A. Shirley, J. Chem. Phys. 64,
3685 (1976).

${ }^{30}$ M. Ohno and G. Wendin, J. Phys. B 12, 1305 (1979).

${ }^{31}$ S. Aksela, M. Kellokumpu, H. Aksela, and J. Väyrynen, Phys. Rev. A 23, 2374 (1981).

${ }^{32}$ W. Mehlhorn, B. Breuckmann, and D. Hausamann, Phys. Scr. 16, 177 (1977).

${ }^{33}$ C. A. Nicolaides and D. R. Beck (unpublished).

${ }^{34}$ M. O. Krause and C. W. Nestor, Phys. Scr. 16, 285 (1977).

${ }^{35}$ B. W. Veal, D. J. Lam, H. Diamond, and H. R. Hoekstra, Phys. Rev. B 15, 2929 (1977). 